\title{
Electroweak Couplings, Lepton Universality, and the Origin of Mass: An Experimental Perspective
}

\author{
John Swain \\ Department of Physics, Northeastern University, Boston, MA 02115 \\ E-mail: 'john. swain@cern.ch'
}

ABstract: A somewhat ecletic selection of some of the material presented in my SILAFAE-III talk is presented here with an emphasis on taus and recent LEP results (updated since the talk) and on discussions about the possible role that gravity in connection with understanding the origin of mass.

\section{Introduction}

Among the most remarkable achievements in recent years has been the construction and verification to very high precision of the so-called Standard Model of the electroweak interactions. Based on the concept of a broken gauge symmetry, the theory has passed all tests with flying colours, and yet remains unsatisfactory in many ways.

One of the most puzzling unexplained features of the Standard Model is the existence of generations of fermions: an apparent copying of the up and down quarks, and the electron and its neutrino at least twice for no obvious reason. Earlier in the days of particle physics it had been hoped that the existence of apparently identical particles with identical gauge couplings constants, but different masses, might shed some light on the origin of mass itself. By now the similarities between the couplings of each generation of particles is now so well-established that this symmetry is essentially built in to the Standard Model and is often almost taken for granted. Restricted to the lepton sector, where precise measurements are easier to make than in the quark sector, one speaks of "lepton universality".

The only precise window on any potential breakdown of this structure is provided by the $\tau$ lepton. Electron-muon universality in the context of the Standard Model is well-established, but it is only recently that exhaustive studies of $\tau$ couplings have been possible. Of the other fermions one might study, the quarks are greatly complicated by the strong interaction, and the neutrinos by their stability and small (and indeed unknown) masses and tiny interaction cross sections.

In my talk I reviewed the rather comprehensive experimental support for this generational universality idea with special emphasis on taus. I then described the status of searches for the Higgs boson, our current best guess about the origin of mass and finally concluded with some speculations on the possible role that gravity may play in understanding mass.

The plan of this writeup is similar. First I review studies of the $\tau$ charged current current couplings from tau decays, searches for anomalous derivative couplings, and then, since much of the best evidence for universality in the Standard Model comes from the studies of the $Z$ at LEP, I summarize the LEP results on $Z$ couplings. I then cover recent experimental results on a search for the Higgs boson, and end with some speculations on gravity.

For more recent updates on studies of Michel parameters and anomalous derivative couplings, which show no signs of deviation from the predictions of universality, I refer the interested reader to the recent review of Rougé[in], and my talk at TAU2000 [3i] (respectively). 


\section{2. $\tau$ Michel Parameters}

Allowing a general combination of local scalar, pseudoscalar, vector, axial vector and tensor interactions, the 4 -fermion interaction describing leptonic tau decays can be specified in terms of 4 Michel parameters $\rho, \eta, \xi, \xi \delta$ (expected to be $3 / 4,0,1,3 / 4$ for the Standard Model). Neglecting radiative corrections, the decay width of a $\tau$ lepton into a charged lepton $\ell$ in solid angle $\Omega$ with respect to the $\tau$ spin is:

$$
\begin{aligned}
\frac{d \Gamma}{d \Omega d x}= & \frac{\mathrm{G}_{\mathrm{F}}^{2} \mathrm{~m}_{\tau}^{5}}{192 \pi^{4}} x^{2}\left[3(1-x)+\frac{2}{3} \rho(4 x-3)+\right. \\
& 6 \eta \frac{\mathrm{m}_{\ell}}{\mathrm{m}_{\tau}} \frac{1-x}{x}- \\
& \left.\xi P_{\tau} \cos \theta\left((1-x)+\frac{2}{3} \delta(4 x-3)\right)\right]
\end{aligned}
$$

where $x$ is the fraction of the maximum possible energy carried off by the lepton $\ell$ and $P_{\tau}$ the tau polarization. Some knowledge of the $\tau$ spin direction is needed to obtain the Michel parameters. Taus are produced with intrinsic polarisation from $Z^{0}$ decays at LEP, but both at LEP and at lower energies an "effectively polarized" sample can be obtained by noting that production of tau pairs from a $J=1$ state forces their spins to be highly correlated. Figure $\underline{1}_{1}^{1}$ shows the results [4it] of fits to the observed tau leptonic decay spectra as of the TAU98 conference. Newer results have since become available and the interested reader is referred to the proceedings of TAU2000, in press at the time of writing.

The best determination of $\eta$ is from total $\tau$

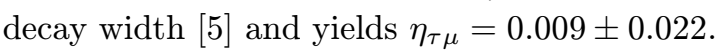

\section{Anomalous Electric and Magnetic Dipole Moments of the $\tau$}

One can look for anomalous couplings of the $\tau$ lepton to the electromagnetic field by parametrizing the coupling of the $\tau$ lepton to photons of 4-momentum $q$, replacing the usual $\gamma_{\mu}$ coupling by

$$
\gamma_{\mu}+i \frac{F_{2}\left(q^{2}\right)}{2 m_{\tau}} \sigma_{\mu \nu} q^{\nu}-F_{3}\left(q^{2}\right) \sigma_{\mu \nu} q^{\nu} \gamma_{5} .
$$

This is the most general form allowed by Lorentz invariance neglecting a possible anapole moment

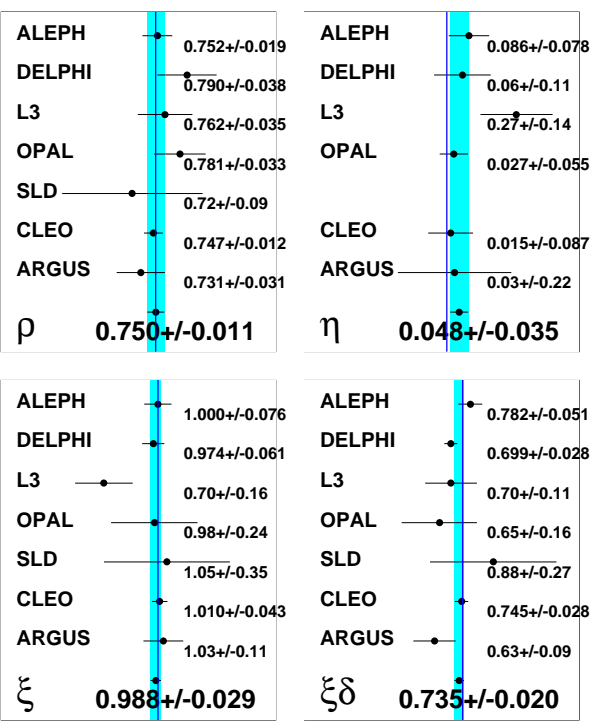

Figure 1: $\tau$ Michel parameters following Stahl.

term and assuming the $\tau$ has charge $e$ 剑. The form factors at $q^{2}=0$ and with the $\tau$ close to mass-shell have special significance : $F_{2}(0)=$ $\frac{g-2}{2}=a_{\tau}$ anomalous magnetic moment of the tau, and $F_{3}(0)$ is the electric dipole moment $d_{\tau}$ of the tau. Hermiticity of the electromagnetic current forces all these form factors to be real, while $\mathrm{T}$ invariance requires that $F_{3}=0$. Upper limits on $F_{2}\left(q^{2}\right)$ for $q^{2} \neq 0$ have been derived from their effect on the tau pair production cross section [的“

The case of $q^{2}=0$ can be studied in radiative tau pair production While $\tau$ 's at LEP are quite clean, the analysis of this data is highly nontrivial, requiring per mil accuracy on signal and background. A comparison of observed data

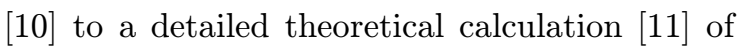
the expected spectrum including all interference and mass terms gives the likelihood distribution shown in figure 2 . If the $\tau$ mass is neglected the large interference between the Standard Model and $F_{2}$ contributions is not included.

The corresponding constraints on anomalous moments from L3 are $a_{\tau}=0.004 \pm 0.027 \pm 0.023$ and $d_{\tau}=(0.0 \pm 1.5 \pm 1.3) \times 10^{-16} e \cdot \mathrm{cm}$.

A similar analysis from OPAL [1 $12 \overline{2}]$ using a simplified calculation of the expected effects of anomalous moments [i $[\overline{1} \overline{3}]$ and a somewhat less powerful analysis technique gives $-0.068<a_{\tau}<$ 


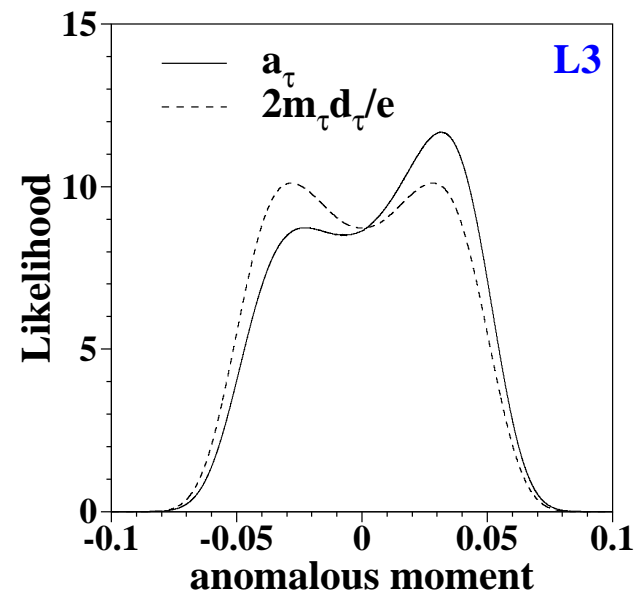

Figure 2: Likelihood distributions for anomalous moment of the $\tau$ from the L3 analysis of $\tau \tau \gamma$ production.

0.065 and $-3.8 \times 10^{-16} e \cdot \mathrm{cm}<d_{\tau}<3.6 \times 10^{-16} e$. $\mathrm{cm}$ at the $95 \%$ confidence level.

\section{Anomalous Weak Neutral Electric and Magnetic Dipole Moments}

By analogy with the electromagnetic case described above, we can add a term coupling the $\tau$ to the $Z^{0}$ gauge boson of the form

$$
i \frac{e a_{\tau}^{W}}{2 m_{\tau}} \sigma_{\mu \nu} q^{\nu}-d_{\tau}^{W} \sigma_{\mu \nu} q^{\nu} \gamma_{5}
$$

to the Standard Model lagrangian and investigate the effects of the parameters $a_{\tau}^{W}$ and $d_{\tau}^{W}$ with tau pairs produced at the $Z^{0}$. The expectations for these quantities in the Standard Model are well below any chance of detectability with present data, but any large observed values would be a definite indication of new physics.

A number of variables can be constructed out of the 4-momenta of the $\tau$ decay products and the $\tau$ flight direction, and comparison with theoretical predictions yields LEP averages [15.1] of $\operatorname{Re}\left(d_{\tau}^{w}\right)\left|<3.0 \times 10^{-18} e \cdot \mathrm{cm}, \operatorname{Im}\left(d_{\tau}^{w}\right)\right|<9.2 \times$ $10^{-18} e \cdot \mathrm{cm}$, and $d_{\tau}^{w} \mid<9.4 \times 10^{-18} e \cdot \mathrm{cm}$.

For effects on single tau polarization, see ref-

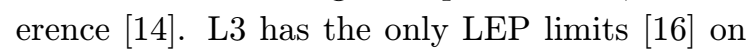
$a_{\tau}^{W}$ and SLD has a preprint [1] its. The combined averages are

$$
\begin{aligned}
\operatorname{Re}\left(a_{\tau}^{W}\right) & =(0.22 \pm 1.1) \times 10^{-3} \\
\operatorname{Im}\left(a_{\tau}^{W}\right) & =(-0.03 \pm 0.66) \times 10^{-3} \\
\operatorname{Re}\left(d_{\tau}^{W}\right) & =(0.09 \pm 0.61) \times 10^{-17} \mathrm{e} \cdot \mathrm{cm} \\
\operatorname{Im}\left(d_{\tau}^{W}\right) & =(-0.13 \pm 0.29) \times 10^{-17} \mathrm{e} \cdot \mathrm{cm}
\end{aligned}
$$

\section{5. $\tau$ Anomalous Weak Charged Elec- tric and Magnetic Dipole Moments}

Following Rizzo [는 what was done in the electromagnetic case, we can modify the Standard Model coupling of the $\tau$ to the $W$ by replacing $\gamma^{\mu}\left(1-\gamma_{5}\right)$ by

$$
\left(\gamma_{\mu}+\frac{i}{2 m_{\tau}} \sigma_{\mu \nu} q^{\nu}\left(\kappa-i \tilde{\kappa} \gamma_{5}\right)\right)\left(1-\gamma_{5}\right)
$$

The best results for these parameters are obtained from examination of their effect on the leptonic $\tau$ decay width [i5] which yields: $\kappa=$ $0.001 \pm 0.008$ and $\tilde{\kappa}=0.00 \pm 0.16$.

These are significantly better than previous results from branching ratios [1] $\left.\bar{L}_{1}^{\prime}\right](|\kappa|<0.0283)$ and an extension of Michel parameter analysis by DELPHI [20"] $(\kappa=0.029 \pm 0.036 \pm 0.018)$. Attempts to use the $\rho$ decay mode of the $\tau$ are more difficult but have been attempted [i1 $\overline{1}_{1}^{\prime}$.

\section{Combined Limits on Derivative Cou- plings}

At the cost of some model-dependence (namely assuming that anomalous couplings have $S U(2)_{L} \times$ $U(1)_{Y}$ symmetry), it is possible to derive better combined limits[2 $\left[21_{1}^{1}\right]-0.007<a_{\gamma}<0.005$ $-0.0024<a_{Z}<.0025$, and $-0.004<\kappa_{W}<.004$ where $2 \sigma$ limits are quoted.

Great care must be exercised in combining values for form factors which are, in general, (modeldependent) functions of the 4-momenta on offshell fermion lines and of $q^{2}$ at the vertex. For this reason we do not attempt to include the re-

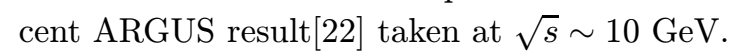

\section{Z couplings}

By studying the relative production probabilities of various particles and their distributions 
in space and momentum, detailed comparisons can be made with the electroweak theory that is meant to describe the $Z^{0}$ boson.

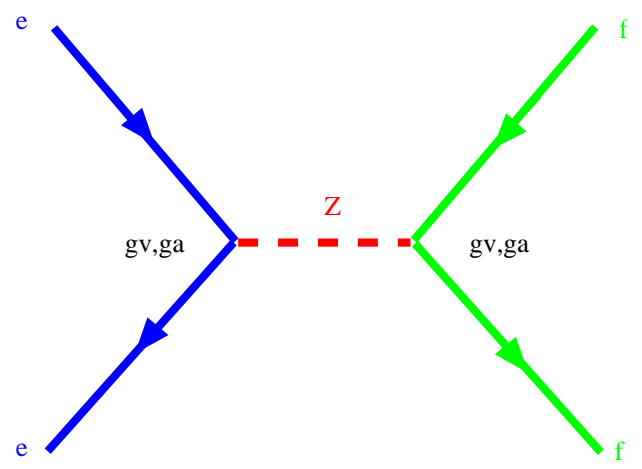

Figure 3: Feynman diagram for production of fermion-antifermionpairs from $e^{+} e^{-}$collisions via the $Z^{0}$.

Fermion pair production via the $Z^{0}$ can be thought of as a superposition of probabilities to produce various combinations of right and left handed fermions and antifermions. The admixtures of right and left are not in general equal, with the discrepancy being parameterized in terms of couplings constants to vector and axial vector pieces of the $Z^{0}, g_{v}$ and $g_{a}$ respectively. The difference between the two corresponds to the lefthanded piece of the interaction, while the sum corresponds to the right-handed piece.

When we discuss the couplings of the $Z^{0}$ to different fermions, it is interesting to relax the standard assumption that these couplings are the same to all fermions, and instead of $g_{v}$ and $g_{a}$, write $v_{f}$ and $a_{f}$ for the respective couplings to fermion $f$.

Various combinations of these can be measured (some much more easily than others) but all provide information on $Z^{0}$ couplings.

The sum total is simply the total cross section, and this can be measured directly. It is proportional to $\left(a_{e}^{2}+v_{e}^{2}\right)\left(a_{f}^{2}+v_{f}^{2}\right)$. An exact expression is difficult to write down in closed form, as radiative corrections both from the initial and final state particles are important. Results on partial widths into leptons are shown in figure

Another important observable at the $Z^{0}$ is the forward-backward asymmetry. The idea here is that we're colliding particles and antiparticles (electrons and positrons) and it's interesting to

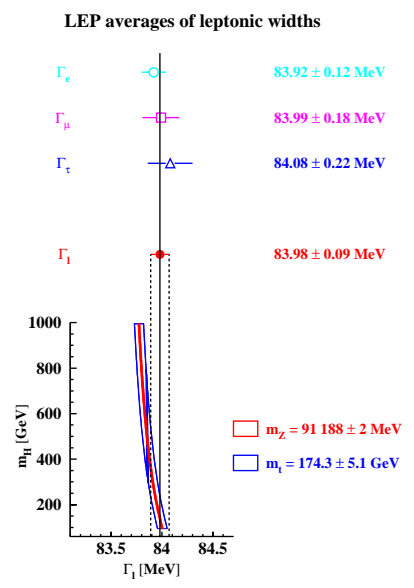

Figure 4: LEP results on the partial widths of the $Z^{0}$ into hadrons and into leptons.

measure whether or not the final state fermions tend to follow the direction of the electrons, or the positrons - as if the "charge" had a sort of momentum associated with it. More formally,

$$
A_{F B}=\frac{\sigma_{F}-\sigma_{B}}{\sigma_{F}+\sigma_{B}}
$$

where the $F$ and $B$ subscripts represent "forward" and "backward" respectively. This quantity turns out to be proportional to $\frac{a_{e} v_{e} a_{f} v_{f}}{\left(a_{e}^{2}+v_{e}^{2}\right)\left(a_{f}^{2}+v_{f}^{2}\right)}$. Note that it suffices to merely determine the charges of the outgoing fermions and into which hemispheres of the detector (defined by a slice through the interaction point and perpendicular to it) the particles went. This measurement is very nice experimentally for a number of reasons, including the fact that measurements of luminosity and absolute detection efficiency cancel in the ratio defining $A_{F B}$.

The results of detailed measurements from all the LEP collaborations are shown in figure $\underline{\bar{s}}_{1}$ for leptons assuming universality (no deviation was observed). The superscript 0 means that the asymmetries have been corrected so that the data is all as if it were taken right on the $Z^{0}$ peak ( $A_{F B}$ depends on the centre-of-mass energy).

The tau lepton is unique among the fermions produced at LEP as its decays can be used to measure its average polarization $P_{\tau}$, giving new and independent information on the $Z^{0}$ couplings apart from the total cross section and forward- 


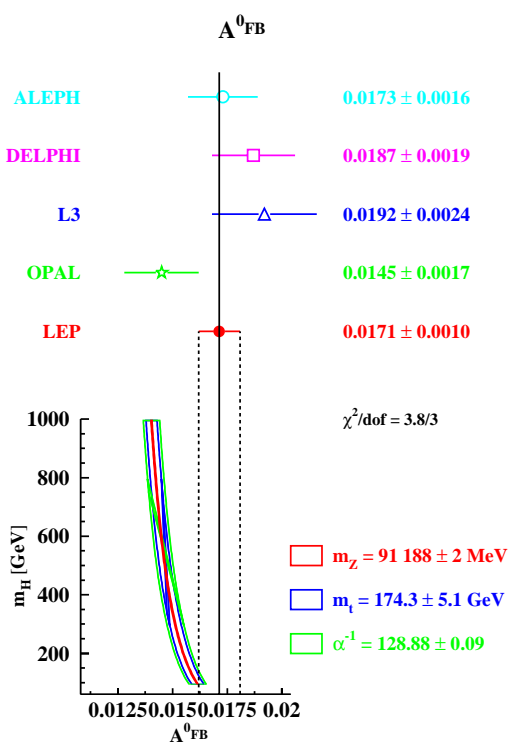

Figure 5: LEP results on $A_{F B}$

backward asymmetry. $P_{\tau}$ is proportional to $\frac{a_{f} v_{f}}{a_{f}^{2}+v_{f}^{2}}$. It is often convenient to defined $A_{\tau}=-P_{\tau}$ for reasons which will become clear later.

The basic idea is simple: the tau decays via the weak interaction which is purely left-handed (V-A). Let's consider the case of the decay $\tau^{-} \rightarrow$ $\pi^{-} \nu_{\tau}$ for concreteness. When the $\tau$ decays, the $\pi^{-}$, being spinless, carries away no helicity. That means that the helicity of the $\tau$ is carried entirely by the neutrino. But the neutrino is always lefthanded (as far as we know), so the direction of the neutrino flight gives the direction of the neutrino spin and thus of the tau spin. Now we can't measure the neutrino, but we do know that the pion had to come off exactly opposite it in the tau rest frame in order to conserve linear momentum. That means that the pion direction tells you the neutrino direction which tells you the neutrino spin which tells you the tau spin the tau reveals its spin direction from the direction in which is spits out the pion - it is its own polarimeter!

At LEP the taus are produced with considerable boosts, so what one measures in practice is the energy distribution of the pion, which tells you if the pion flew off with or against the direction of motion of the tau which we can take as a direction to define helicity. Arguments similar to the one in the previous paragraph show that the other decay modes of the $\tau$ also contain information about its polarization, though always a bit more diluted. In the cases of tau decays to muons or electrons one loses two neutrinos which decreases the sensitivity significantly, and in the case of the $\rho$, the fact that it is a vector particle means that it has two possible ways to come off (helicity 1 or helicity 0) and still conserve angular momentum. Sensitivity can be regained by using the distributions of the two pions from the $\rho$ decay to help analyze the spin of the $\rho$ itself. When all factors are taken into account, the $\rho$ decay mode turns out to have the largest statistical weight.

Of course we have to use the assumed V-A structure of the charged weak interactions as a polarimeter, so this may look a bit like a swindle - we're using part of a theory to test the theory itself! There is actually a way to check the V-A assumption in tau decays and this is using the decay $\tau \rightarrow a_{1} \nu_{\tau}$ where the $a_{1}$ decays into three pions. This decay is interesting since it is dominated by $\rho \pi$ but there are in fact two ways to make a $\rho$ in the final state and the amplitudes for these two possibilities must be added quantum mechanically. The resulting interference is actually sensitive to the absolute sign of the neutrino helicity (i.e. can distinguish between $\mathrm{V}-\mathrm{A}$ and $\mathrm{V}+\mathrm{A}$ ) and the assumption of $\mathrm{V}$-A turns out to hold very well indeed! Possible admixtures of scalar, pseudoscalar and tensor interactions in the decay amplitude are strongly constrained by measurements of the Michel parameters, discussed above.

One final thing we can do with taus is to ask whether the polarization itself (we've looked at the average so far) has an asymmetry - in other words, does one tend to produce more right handed taus that go in the direction the electron went, or opposite to it? To this end we defined $A_{\text {pol }}^{F B}$ which is approximately $-\frac{3}{2} \frac{a_{e} v_{e}}{a_{e}^{2}+v_{e}^{2}}$. This can be written as $\frac{3}{2} A_{e}$ where $A_{e}$ is just like the $A_{\tau}$ we defined above, but with tau couplings replaced by electron couplings. Note that this quantity depends not on couplings of the $Z^{0}$ to the tau, but to the electron!

The results of detailed measurements from 

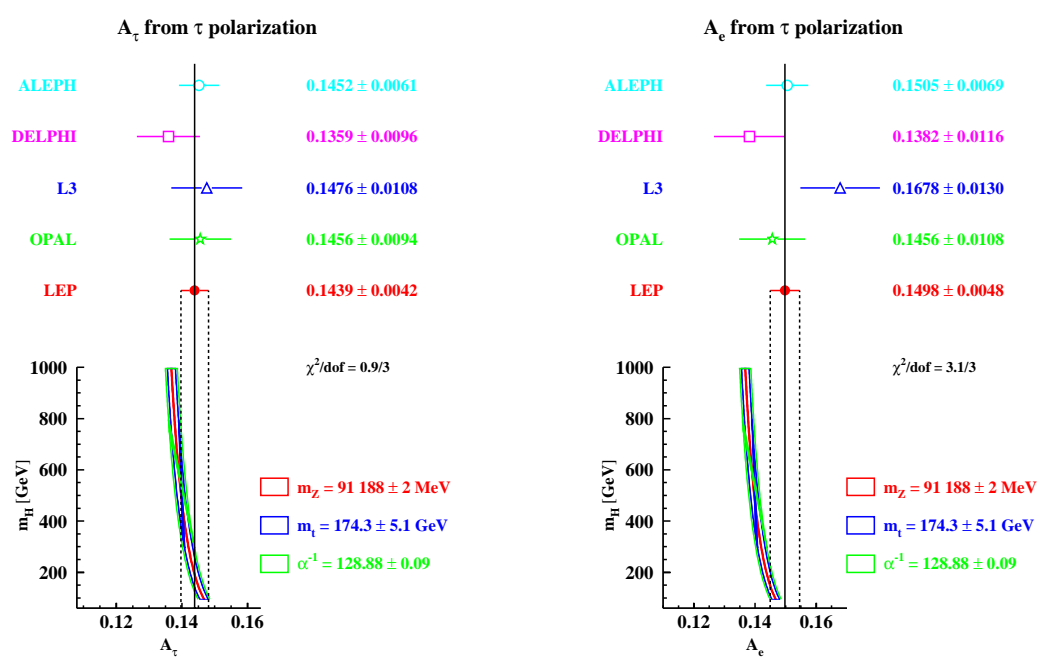

Figure 6: LEP results on tau polarization and polarization asymmetry.

all the LEP collaborations are shown in figure

A fit to the total LEP data sample gives values of the vector and axial vector couplings constant $g_{v}$ and $g_{a}$ shown in figure $\bar{i}$. Note that the largest errors are for the muons, since the tau analyses provide additional information on both $\tau$ and electron couplings. Arrows and a distorted rectangle indicate how the values would change with changes in the Higgs and top mass from the fitted values. The ellipse referred to as "combined" indicates the results if lepton universality is assumed (i.e. that the electroweak couplings are the same to each lepton). The band shows the results from SLD on the same quantities using the left-right asymmetry, an additional asymmetry which is only accessible to that experiment since SLC provides (longitudinally) polarized beams.

Leptonic branching ratios of the $W^{ \pm}$turn out to be just as expected from the Standard Model as shown in figure $\overline{\bar{p}_{r}}$

\section{The Higgs Boson}

The Higgs boson sector in the Standard Model remains largely unknown and the explanation of mass as a coupling to this field sheds no light at all on the existence of generations. The fact that the Higgs boson is meant to couple to mass means that the chance of producing it in $e^{+} e^{-}$

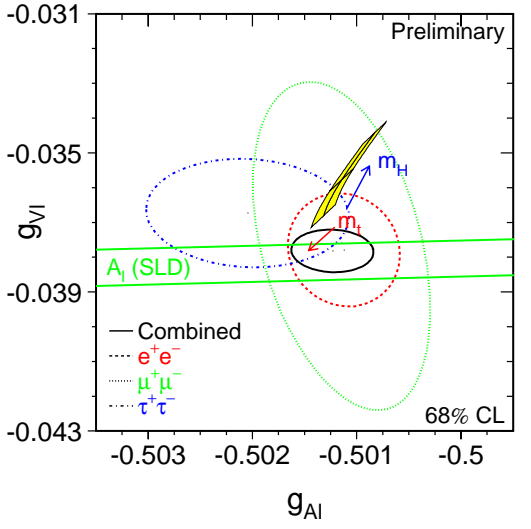

Figure 7: LEP results on $g_{a}$ and $g_{v}$.

collisions in the s-channel like the $Z^{0}$ is hopeless. The Higgs boson can be looked for at LEP in two different ways:

- Direct production, where it is radiated from a (heavy!) $Z^{0}$ boson and decays into (heavy!) b quarks, or can be reconstructed from the invariant mass of the $Z$ decay products.

- From its contributions to radiative corrections where it appears in loop diagrams. While the use of radiative corrections to see virtual particles and probe beyond the actual energy available at LEP has been very successful in the case of the top quark, for 
Summer 00 - Preliminary - [161-207] GeV

W Leptonic Branching Ratios

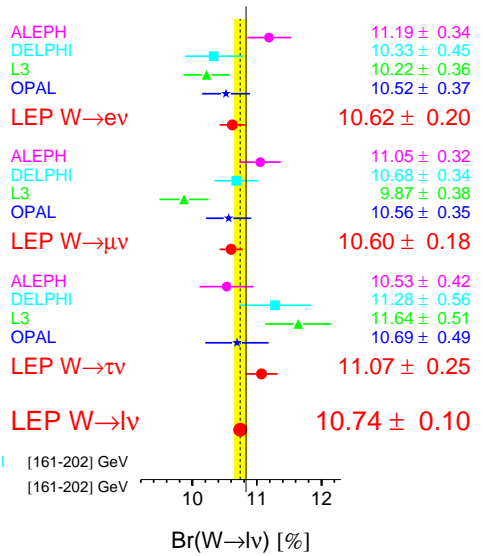

Figure 8: LEP results on $W$ branching ratios.

which accurate determinations of its mass have been made at LEP without being able to make a single one on-shell, the Higgs is much more problematic. Its mass generally appears only logarithmically and thus radiative corrections are only weakly sensitive to it.

The status of searches for a Higgs boson are summarized in figures $\overline{\underline{9}}_{1}^{\overline{1}}$ and $\overline{1}_{1}^{1} \underline{0}_{\mathrm{r}}^{\prime}$. Shaded regions show exclusions from direct searches while ovals or curves show the results of fits to electroweak data.

\section{The CKM Matrix}

Space considerations do not allow any reasonable treatment of the CKM matrix, but I would like to offer a few points here for consideration. First of all, the CKM matrix is nothing more than our way of parameterizing the fact that the weak eigenstates need not be mass eigenstates. Again we see the problem of mass appearing, but in a rather subtle way: not only do we have to give (sharp) masses to some states, but we have to understand which particular states get sharp masses - what picks out the preferred basis for mass eigenstates?
One of the main benefits of the CKM matrix is that it naturally contains one complex phase which cannot be reparametrized away, and may be ultimately responsible for $\mathrm{CP}$ violation. This is not the case if a quark mass were to be zero, so here we see that even questions such as $\mathrm{CP}$ violation ultimately depend at some point on mass.

In the neutrino sector it is also possible that a mixing matrix is present. Again, the question of whether neutrinos have mass or not is crucial. With recent suggestions that neutrinos may have masses, it is all the more painfully apparent that we've not really got a very good theory of mass: not only do we not know any masses or mixing angles ahead of measuring them, we don't even know if any of them are nonzero, or even of one solid relation between them!

\section{Mass and Gravity?}

While in the Standard Model mass is just a coupling to some sort of omnipresent scalar field, if one asks beginning physics students what mass is, one invariably winds up getting some suggestion that it might have something to do with gravity.

In the Standard Model, gravity is left out completely, the idea being that it is usually negligible and can be added in at some point later on if need be, perhaps as some sort of (weak) perturbation. There are, in fact, various reasons to think that gravity may play an important role and that the Higgs mechanism and in fact the whole renormalization program is really just a stop-gap or mathematical trick that allows us to do calculations. In this section I'd just like to make a few comments along these lines ... it's perhaps more interesting to be left with questions than given answers!

Perhaps the oldest suggestion that gravity might have something to do with mass is that of Mach [24]. Suppose that the universe were completely empty - how would one know if a lone object were accelerated or not? With respect to what would one say it were accelerated? Usually one thinks of inertial frames as being unaccelerated with respect to the "distant stars" - but what if they weren't there? 

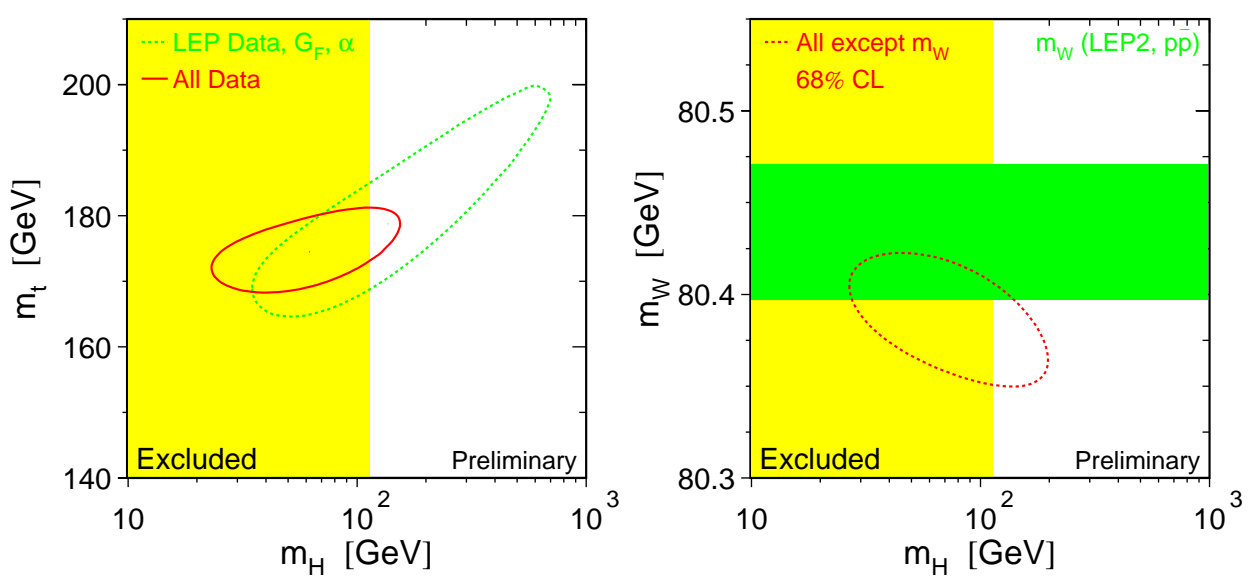

Figure 9: LEP results on Higgs mass versus top and W masses.

In Mach's own words, "But what would become of the law of inertia if the whole of the heavens began to move and the stars swarmed in confusion? How would we apply it then? How would it have to be expressed then?"

If the inertial properties of objects were due to gravitational interactions with distant matter in the universe that would help us understand the fact that inertial and gravitational mass are the same - things are hard to accelerate because the rest of the mass in the universe tries to hold them in place gravitationally! Of course the Higgs mechanism has nothing to do with Mach's principle.

Let's have a look at another place where gravity may have something to do with mass. An interesting toy model to play with is a spherical shell of radius $r$ with a charge $e$ uniformly distributed on it. As the radius is taken to zero, the electromagnetic self-energy diverges as $1 / r$ and this is usually taken to indicate some sort of pathology in the theory at short distances. Now let's try to do the calculation including gravity. I learned about this from $\overline{2} \overline{5}$.

The total mass (take $c=1$ ) at finite radius $r$ is that due to the bare mass $m_{0}$ of the shell itself, plus the electromagnetic self energy $e^{2} / r$.

$$
m(r)=m_{0}+\frac{e^{2}}{r}
$$

Now let's add the gravitational self-energy.

$$
m(r)=m_{0}+\frac{e^{2}}{r}-G \frac{m_{0}^{2}}{r}
$$

Note that while the charge tends to expand the sphere, gravity tends to shrink it. This is, however, not really any better since depending on the overall sign of the quantity multiplying $\frac{1}{r}$, this still blows up either negatively or positively as the radius tends to zero.

But shouldn't all energy gravitate? Taking a hint from general relativity, let's write instead

$$
m(r)=m_{0}+\frac{e^{2}}{r}-G \frac{m(r)^{2}}{r}
$$

This may seem like a small change, but what has happened now is that we've got a quadratic equation for $m(r)$ which can be easily solved for any $r$. The amazing thing now is that $m(r)$ has a finite limiting value of $\frac{e}{\sqrt{G}}$ as $r$ goes to zero! Not only that, but this is independent of the bare mass $m_{0}$.

Before you dismiss this as a naive toy, let me point out that a careful treatment in the context of general relativity also gets you a finite result in other words, gravity doesn't make "ultraviolet divergences" worse; it makes them better!

Note that the result is also non-perturbative in $G$ - there is no convergent power series for $\frac{1}{\sqrt{G}}$ about $G=0$. It may amuse you to have a look at what goes wrong if you try to approximate the 

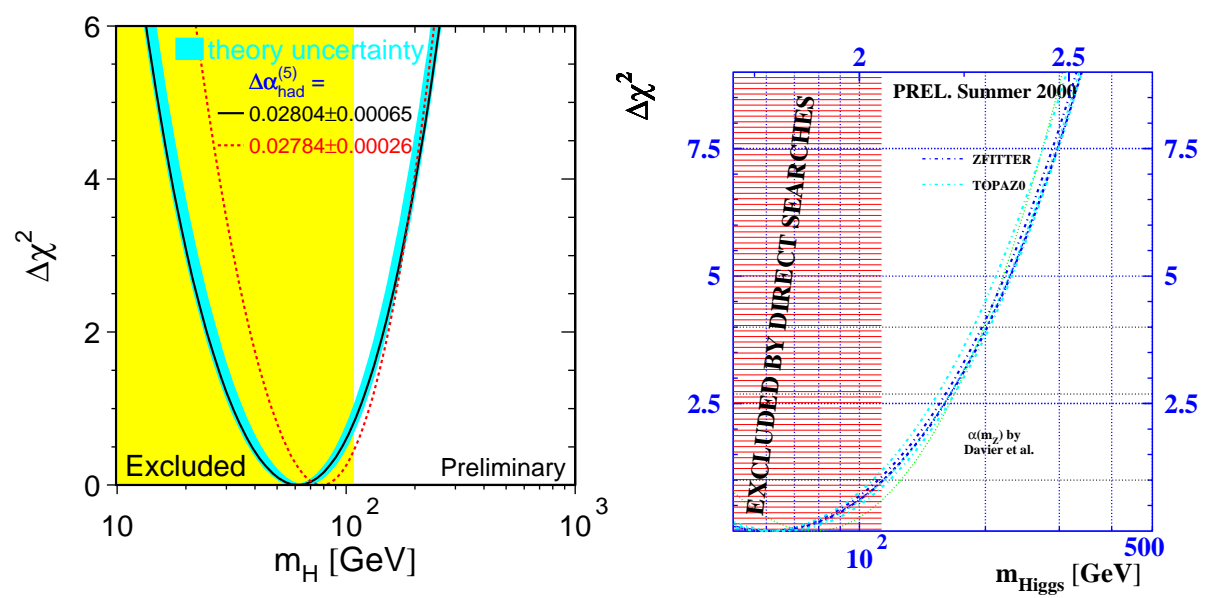

Figure 10: LEP results on Higgs mass. Note that the direct searches already rule out the value of Higgs mass most favoured from radiative corrections, although the errors are still very large.

quadratic equation solution above with a series in $G$.

Suggestions that similar things are true in quantum corrections to the self-energy of a charged scalar particle have also been known for decades [26], though this line of investigation has not been followed much in recent years.

The warning, it seems, is that gravity may be deeply related to mass and it knows how to get finite answers out of otherwise hopeless-looking cases. However, it looks like it must be included from the outset and adding it in at the end of the day as a small correction is unlikely to do much good.

Incidentally, the whole (rather successful, it must be noted!) renormalization program calculates infinite corrections to particle masses and then forces them by hand to agree with the observed masses. Such an approach, it seems to me, has little hope of predicting or explaining any masses.

One final calculation which may amuse you is one of the width for a Higgs decay into gravitons [2] $[2]$. Before I sketch this, let's be perfectly up-front about how dangerous it is to mix Higgs physics with general relativity. The scalar field corresponds to an enormous vacuum energy density which ought to curl the universe up into a small sphere which is wildly in disagreement with observation - you have to assume that the Higgs field doesn't do anything gravitationally which sort of flies in the face of the equivalence principle. Nevertheless, let's see what one might try to calculate.

Einstein tells us that (Ricci and scalar) curvature is given by the stress-energy-momentum tensor.

$$
R_{\mu \nu}-\frac{1}{2} g_{\mu \nu} R=\frac{8 \pi G}{c^{4}} T_{\mu \nu}
$$

The Higgs field is supposed to be a scalar field that couples to mass, or, to put it a bit more relativistically, the trace of $T$. Now $T$ is proportional to $R$, so we should get a coupling of the scalar field to $R$. If you put this all together, you find that $G$ drops out (the equivalence principle at work $)$ and you find $\Gamma(H \rightarrow$ $g g)=\frac{\sqrt{2}}{16 \pi} \frac{G_{F} M_{H}^{2}}{\hbar c} \frac{M_{H} c^{2}}{\hbar}$ which is about a million times bigger than the width for $\Gamma(H \rightarrow \gamma \gamma)^{1}$. Can one really forget about gravity when one is thinking about mass?

\section{Acknowledgements}

I would like to thank the organizers of the conference, especially Enrico Nardi, for a most stimu-

\footnotetext{
${ }^{1}$ The two-photon decay mode occurs at one loop, while the gravitational one is already present at tree level. Forgetting this fact seems to have caused some confusion to some people!
} 
lating meeting in beautiful surroundings. I would also like to thank my colleagues at Northeastern University and on LEP and CMS, and the National Science Foundation for its continuous support. LEP results quoted are the most recent ones from the LEP Electroweak Working Group [20 $\left[\overline{8}^{\prime}\right]$ at the time of writing and available at http://lepewwg.web.cern.ch/LEPEWWG.

\section{References}

[1] Good general references for LEP are the following CERN "Yellow Reports": "Z Physics at LEP1", CERN 89-08, 3 volumes, eds. G. Altarelli, R. Kleiss, and C. Verzegnassi; and "Physics at LEP2", CERN 96-01, 2 volumes, eds. G. Altarelli, T. Sjöstrand, and F. Zwirner.

[2] A. Rougé, "Tau lepton Michel parameters and new physics", http://xxx.lanl.gov, hep-ph/0010005.

[3] J. Swain, "Anomalous Electroweak Couplings of the Tau and Tau Neutrino", talk at the Sixth International Workshop on Tau Lepton Physics (TAU2000), Victoria, British Columbia, Canada, September 18-21, 2000, to appear in the Proceedings.

[4] A. Stahl, "Michel Parameters. Averages and Interpretations", Proceedings of TAU98, Nucl. Phys. B, Proc. Suppl. vol 76.

[5] M.-T. Dova, J. Swain and L. Taylor, Phys.Rev.D58:015005,1998, "Constraints on anomalous charged current couplings, tau neutrino mass and fourth generation mixing from tau leptonic branching fractions", Proceedings of TAU98, op. cit.

[6] M. C. Gonzalez-Garcia, A. Gusso, and F. Novaes, eprint hep-ph/9802254.

[7] W. J. Marciano, Proceedings BNL Summer Study on CP Violation, Brookhaven National Laboratory, eds. S. Dawson and A. Soni, 1990.

[8] D. J. Silverman and G. L. Shaw, Phys. Rev. D27 (1983) 1196;

R. Escribano and E. Massó, Phys. Lett. B301 (1993) 419;

R. Escribano and E. Massó, Nucl. Phys. B429 (1994) 19;

G. Köpp, D. Schaile, M. Spira, and P. M. Zerwas, Z. Phys. C65 (1995) 545.

[9] J. A. Grifols and A. Méndez, Phys. Lett. B255 (1991) 611, erratum ibid B259 (1991) 512.
[10] L3 Collaboration (M. Acciarri et al.), Phys. Lett. B434 (1998) 169-179.

[11] S.S. Gau, T. Paul, J. Swain, and L. Taylor, Nucl. Phys. B523 (1998) 439.

[12] OPAL Collaboration (K. Ackerstaff et al.), Phys. Lett. B431 (1998) 188.

[13] J. Biebel and T. Riemann, Z. Phys. C76 (1997) 53.

[14] J. Vidal, J. Bernabéu, and G. GonzálezSpringberg, " $\tau$ Weak Dipole Moments from Azimuthal Asymmetries", Proceedings of TAU98, op. cit.

[15] A. Zalite, "LEP Summary on Weak Dipole Moments of the $\tau$ Lepton", Proceedings of TAU98, op. cit.

[16] L3 Collaboration (M. Acciarri et al.), Phys. Lett. B426 (1998) 207.

[17] SLD Collab., SLAC-PUB-8163 (July 1999).

[18] T. G. Rizzo, Phys. Rev. D56 (1997) 3074.

[19] M. T. Dova, P. Lacentre, J. Swain, and L. Taylor, JHEP 9907 (1999) 026.

[20] P. Seager, "Michel parameters and limits on tensor couplings from DELPHI", Proceedings of TAU98, op. cit.

[21] G. A. González-Springberg, A. Santamaria, and J. Vidal, Nucl. Phys. B582 (2000) 3.

[22] ARGUS Collab., H. Albrecht et al., http://xxx.lanl.gov, hep-ex/0004031.

[23] A. Connes, "Noncommutative Geometry", Academic Press, 1994.

[24] A good reference is J. Barbor and H. Pfister (eds.), "Mach's Principle: From Newton's Bucket to Quantum Gravity (Einstein Studies, Vol 6)", Springer Verlag, 1995.

[25] A. Ashtekar, "Lectures on Non-Perturbative Canonical Gravity", World Scientific, 1991.

[26] B. S. DeWitt, Phys. Rev. Lett. 13 (1964) 114.

[27] Y. Srivastava and A. Widom, http://xxx.lanl.gov, hep-ph/0003311.

[28] http://lepewwg. web.cern.ch/LEPEWWG 